\title{
TECNOLOGIA DA INFORMAÇÃO, SISTEMAS DE INFORMAÇÕES GERENCIAIS E GESTÃO DO CONHECIMENTO COM VISTAS À CRIAÇÃO DE VANTAGENS
}

\author{
COMPETITIVAS: REVISÃO DE LITERATURA
}

Information Technology, Management Information Systems and Knowledge Management

with Views to the Creation of Competitive Advantages: literature review

\author{
Jhony Pereira Moraes ${ }^{1}$ \\ Sidimar Meira Sagaz ${ }^{2}$ \\ Geneia Lucas dos Santos ${ }^{3}$ \\ Deison Alencar Lucietto ${ }^{4}$ \\ Recebido em: 09 jul. 2017 \\ Aceito em: 10 abr. 2018
}

\begin{abstract}
Resumo: Este artigo teve por objetivo descrever usos e aplicações de três ferramentas de gestão presentes no ambiente empresarial contemporâneo: a Tecnologia da Informação (TI), os Sistemas de Informações Gerenciais (SIG) e a Gestão do Conhecimento (GC). Foi realizada revisão narrativa de literatura. Verificou-se que o fluxo de informações funciona como o elemento unificador entre TI, SIG e a GC. Ao possibilitarem o uso adequado de informações e de pessoas com vistas ao alcance dos objetivos organizacionais, fomentam a criação de vantagens competitivas. Identificou-se, então, que a introdução dessas ferramentas, ao alterar processos internos e externos, contribui para o desenvolvimento das organizações.

Palavras-Chave: Tecnologia da Informação. Sistemas de Informações Gerenciais. Gestão do Conhecimento. Vantagem Competitiva.
\end{abstract}

Abstract: This article aims to describe uses and applications of three management tools present in the contemporary business environment: Information Technology (IT), Information Systems Management (ISM) and Knowledge Management (KM). A narrative review of the literature was performed. It was verified that the information flow works as the unifying element between IT, ISM and KM. By enabling the proper use of

\footnotetext{
${ }^{1}$ Mestrando em Administração (UFRGS), Pós-graduando em Docência no Ensino Superior (Centro Universitário Leonardo da Vinci) e graduando em Administração de Empresas (UniRitter). E-mail: jhonymoraes@hotmail.com.br.

2 Mestrando em Administração (UFRGS), Pós-graduando em Marketing (IMED) e graduado em Publicidade e Propaganda (UNOCHAPECÓ). E-mail: sidimarsagaz@gmail.com.

${ }^{3}$ Doutoranda em Administração (UFRGS), Mestra em Administração (UNISUL), graduação em Psicologia (UNIARP) e Administração (UNC). E-mail: geneialucas@gmail.com.

${ }^{4}$ Doutor em Saúde Pública (FIOCRUZ), Mestre em Saúde Pública (FIOCRUZ) e graduação em Odontologia (UFSM). E-mail: deisonlucietto@hotmail.com.
} 
information and people to achieve the organizational objectives, they promote the creation of competitive advantages. It was identified, then, that the introduction of these tools, by altering internal and external processes, contributes to the development of organizations.

Keywords: Information Technology. Management Information Systems. Knowledge management. Competitive advantage.

\section{INTRODUÇÃO}

Para que empresas possam se desenvolver e atuar ativamente frente à concorrência, ampliando clientes e mercados, necessitam de informações qualificadas. O investimento em ferramentas tecnológicas de cunho informacional se faz necessário para que os processos de tomada de decisão levem ao alcance dos objetivos empresariais.

O emprego da Tecnologia da Informação ( $\mathrm{TI}$ ) envolve o uso de computadores, tecnologias de comunicações, hardwares e serviços para aprimorar operações, processos e promover transformações de diversas ordens (LAURINDO et al, 2001). Os Sistemas de Informações Gerenciais (SIG), por sua vez, facilitam a tomada de decisões dos administradores (executivos), a partir de dados atualizados, dentro de uma perspectiva global (CARMO; PONTES, 1999). Já, a Gestão do Conhecimento (GC) torna-se essencial para o adequado manuseio da tecnologia com vistas à estratégia de mercado e à organização interna da empresa. Ela busca compreender as características do ambiente competitivo e as necessidades coletivas e individuais da organização, refletindo a coordenação de esforços em nível operacional e estratégico (TERRA, 2014).

Diante do exposto, este estudo teve como objetivo verificar usos e aplicações da TI, SIG e GC enquanto ferramentas de gestão no ambiente empresarial contemporâneo visando à criação de vantagens competitivas. Ele justifica-se na medida que ainda existem dúvidas, no âmbito da gestão, sobre seus alcances e possibilidades com vistas ao aprimoramento dos processos gerenciais.

\section{DESENVOLVIMENTO}

Nesta sessão procurou-se explanar de maneira geral os temas centrais da revisão de literatura.

MÉTODO

Tratou-se de pesquisa bibliográfica do tipo revisão narrativa de literatura, considerando seu uso como adequado "para descrever e discutir o desenvolvimento ou o 'estado da arte' de um determinado assunto, sob ponto de vista teórico ou contextual" 
(ROTHER, 2007, p. 05).

Foram analisados dados de fontes bibliográficas (GIL, 2010) coletados em fontes de informações como livros de referência e artigos científicos, buscando-se por definições, usos, aplicações, vantagens e desvantagens da TI, dos SIG e da GC tendo em vista a criação de vantagens competitivas. A revisão foi conduzida a partir de experiências e aprendizados prévios (MINAYO, 2008).

\section{VANTAGEM COMPETITIVA}

Os primeiros relatos sobre vantagem competitiva derivam dos anos 70, onde o avanço da indústria japonesa suscitou a preocupação das organizações pela definição de um planejamento estratégico, observando o cenário competitivo que se constituía (BRITO; BRITO, 2012). Historicamente, o modelo conceitual mais difundido na área foi (e continua sendo) o New Industrial Organization (Nova Organização Industrial), que aborda a performance das empresas. Segundo essa concepção, as empresas devem ser estratégicas quanto às negociações, em referência a preços, publicidade, investimento, pesquisa e desenvolvimento, o que influencia o posicionamento no mercado competidor. Para isso, é essencial a construção de altas barreiras de entrada no mercado, entendendo os monopólios ou oligopólios como estruturas que maximizam a atratividade das firmas e evitam a queda da lucratividade (PORTER, 1989; VASCONCELOS; CYRINO, 2000).

Dentro da nova organização industrial é evidenciado outro modelo, denominado estrutura-comportamento-performance. Neste modelo a performance da firma é resultante do comportamento da concorrência em termos de preços e custos, que variam conforme o comportamento da indústria. Contudo, o comportamento concorrente não o único balizador, é necessário que a firma, em sua estratégia competitiva, encontre uma posição na estrutura da indústria que a possibilite desenvolver suas forças competitivas (VIANA et al., 2016).

Além disso, Viana et al. (2016) trazem um panorama histórico sobre vantagem competitiva enquanto elemento dinâmico: a escola austríaca retrata a firma como uma estrutura de difícil imitação; alicerçada em um posicionamento inovador, com arranjos econômicos diferentes e que por isso impedem a fácil imitação pela concorrência. Já a teoria das capacidades dinâmicas estuda as "relações entre processos de decisão, as ações empreendidas e suas consequências gerenciais, em termo de formação, conservação e destruição de recursos" (VIANA et al., 2016, p. 536).

As diferenças de performances entre as empresas são originárias do entendimento de que os seus recursos são heterogêneos, o que limita a imitação pela concorrência, observandose que as condições naturais, legais, institucionais, econômicos e organizacionais são pertinentes a realidade de cada uma (BRITO; BRITO, 2012; VASCONCELOS; CYRINO, 2000).

O termo vantagem competitiva é estritamente associado ao aporte financeiro da 
organização, como significado de desempenho superior em relação à concorrência. Contudo, a representatividade do termo está além das finanças, envolvendo, portanto, a geração de valor percebida por clientes e fornecedores através de benefícios oferecidos, do poder de barganha e do próprio relacionamento cliente-empresa (BARNEY, 1991; BRITO; BRITO, 2012; PORTER, 1985). Sendo assim, algumas das premissas da vantagem competitiva são: menor custo de produção, habilidade de provisão e a maximização de benefícios percebidos pelo público atendido (CONTO; ANTUNES JÚNIOR; VACCARO, 2016; VIANA et al., 2016). Além disso, ela necessita ser sustentável e não permitir que outras vantagens competitivas substitutas estejam disponíveis prontamente aos concorrentes (PORTER, 1989).

A geração de valor mencionada, com vistas à satisfação do cliente e melhor posicionamento mercadológico da organização, implica entender de onde esse valor é derivado (ITO et al., 2012). O valor é resultado dos atributos do produto ou serviço (funcionalidade, qualidade, preço e tempo), somado à imagem da empresa e seu relacionamento com as partes interessadas (ITO et al., 2012). Assim, as empresas que geram mais valor para seus clientes detêm vantagens competitivas sobre seus concorrentes (ALMEIDA; MARCONDES, 2014; GHEMAWAT, 2007).

Por fim, entende-se que a vantagem competitiva é relacionada às respostas adaptativas das organizações ao ambiente em que elas estão inseridas e das configurações específicas de competências e recursos valiosos, raros, inegociáveis e insubstituíveis que as mesmas possuem (ALVARENGA et al., 2015; BARNEY, 1991; NOBRE; TOBIAS; WALKER, 2011). Neste sentido, as empresas devem investigar o ambiente de atuação, conferindo as oportunidades e as possibilidades de riscos, podendo-se, então, estabelecer estratégias de curto, médio e de longo prazo - em todos os níveis e estruturas (departamental, humana e informacional) (NOBRE; TOBIAS; WALKER, 2011; VALENTIM, 2003).

\section{TECNOLOGIA DA INFORMAÇÃO}

A TI envolve o uso de computadores, tecnologias de comunicação, hardwares e serviços (LAURINDO et al., 2001). O seu desenvolvimento, a partir dos anos 1950, tem promovido transformações na forma como empresas são operadas (MCGEE; PRUSAK, 1994 apud MORAES et al., 2004). Embora, no passado, a TI fosse utilizada apenas para controlar custos de produção em empresas industriais, hoje possibilita a integração em nível mundial a partir da elaboração de novas estratégias e maneiras de relacionamento entre as empresas e seus consumidores (SILVA, 2002). Compreende-se que a TI visa o aumento da produtividade, bem como a melhoria na qualidade das tomadas de decisões pelos gestores (CINTRA et al., 2012).

De acordo com Rodrigues e Pinheiro (2005) a TI tem usos diversos: 1) possibilita a transformação de dados em informações confiáveis e atualizadas (com vistas à solução de 
problemas e à tomada de decisões); 2) redesenha a estrutura da organização; 3) reduz custos; e 4) promove o uso coletivo de conhecimentos, tecnologias e meios de produção. A segurança em TI pode trazer benefícios, como a transformação no trabalho dos indivíduos, o desempenho da organização e maior produtividade, agindo para a vitalidade da empresa (PRATES; OSPINA, 2004). Por conseguinte, influência nos resultados econômico-financeiros da organização (SILVA, 2002).

Transformações nas empresas derivadas da TI "induzem novos processos e instrumentos que atingem por completo a estrutura e o comportamento das organizações, repercutindo diretamente em sua gestão" (PRATES; OSPINA, 2004, p. 12). Nessa perspectiva, a TI possibilita melhoria dos processos, reduz tempo e espaço, integra unidades de negócio e desenvolve novos perfis de gestão, podendo contribuir na qualidade, produção, análise de mercados e na comunicação com clientes e competidores (ROSSETTI; MORALES, 2007). Neste sentido, pode-se afirmar que a TI está sendo percebida como importante aliada na busca do sucesso organizacional, uma vez que possibilita crescimento e sustentabilidade empresarial (DE HAES; VAN GREMBERGEN; DEBRECENY, 2013; DEBRECENY; GRAY, 2013).

Contudo, o fato de existir TI no ambiente empresarial não é suficiente para garantir a obtenção de vantagens competitivas. Pode-se dizer que fatores como falta de conhecimento, baixa capacitação técnica, investimentos iniciais elevados e infraestrutura falha dificultam a eficiência da tecnologia (OLIVEIRA; BERTUCCI, 2003). Assim, são consideradas dificuldades para a sua operacionalização a complexidade provocada pela inovação em tecnologia e aquela oriunda do relacionamento entre ambientes externo, interno e mercados, os quais demandam acompanhamento criterioso de necessidades de clientes, mudanças de hábitos e sensibilidade de gestores, tendo em mente a instabilidade de mercados (RODRIGUES; PINHEIRO, 2005).

Em meio a tais aspectos, Júnior et al. (2005) ressaltam que é necessário haver sinergia e interação entre o planejamento estratégico e de TI nas organizações. É preciso, então, que exista interação, sinergismo e coordenação na empresa ao longo do tempo (LAURINDO et al., 2001; LUZ et al., 2016). Por conseguinte, as organizações devem estar cientes de que os benefícios reais da TI virão a médio e a longo prazo (MORAES et al., 2004).

\section{SISTEMAS DE INFORMAÇÕES GERENCIAIS (SIG)}

O SIG resulta da implementação da TI através do uso de computadores e telecomunicações (BALARINE, 2002), fornecendo "conceitos, metodologias, técnicas e ferramentas para os executivos das organizações tomarem decisões baseadas em informações estratégicas, precisas, atualizadas e em tempo hábil" (CARMO; PONTES, 1999, p. 49). Para tanto, organizam e projetam bancos de dados de macro abrangência (Contabilidade, Recursos Humanos, Finanças etc.) em informações relevantes para as tomadas de decisão, oferecendo detalhes sobre as operações da empresa (BAZZOTTI, GARCIA, 2006; OLIVEIRA, 2002; STAIR, 
1998). Dentre os SIG estão o ERP (Enterprise Resource Planning), o Data Mining, o Data Warehouse e o CRM (Costumer Relationship Management) (VALENTIM, 2003).

Os SIG auxiliam na coleta, processamento, armazenamento, análise e disseminação de informações de forma a atender propósitos específicos do negócio, subsidiando-se de dados e instruções (entradas) e, posteriormente, de relatórios e cálculos (saídas). Assim, contribuem para a sobrevivência das organizações (AUDY, BECKER, FREITAS, 2014; PEREZ, 2006).

Eles possibilitam aos seus usuários atividades de planejamento e desenvolvimento, como também a proposição de soluções para problemas, sejam eles operacionais, gerenciais, estratégicos (REZENDE; ABREU, 2006).

São benefícios dos SIG aos negócios: 1) elevam os níveis de eficácia e produtividade, gerando soluções e valorizando o uso do conhecimento e da informação (BAZZOTTI; GARCIA, 2006); 2) alinham as estratégias de negócios e de TI, instigando o reconhecimento recíproco das potencialidades de suas respectivas áreas entre gestores e profissionais de TI (ALBERTIN, 1996); 3) reduzem custos de operação (OLIVEIRA, 2002); 4) melhoram o processo produtivo, o fluxo de informações, os serviços oferecidos e a tomada de decisão (OLIVEIRA, 2002).

Entretanto, a implementação de um SIG pode apresentar algumas incongruências. Se, por um lado, ele encurta as distâncias globais entre compradores e vendedores, por outro, demanda investimentos contínuos em atualização e manutenção, podendo, ainda, envolver problemas de distribuição (BALARINE, 2002).

Mesmo diante de possíveis dificuldades, a implementação de um SIG simboliza, entre outros aspectos, a valorização do capital humano e intelectual da empresa, como também a sistematização de informações relevantes para a tomada de decisão em todos os níveis organizacionais (REZENDE; ABREU, 2000). Assim, por meio dos SIG as empresas são capazes de desenvolver vantagem competitiva e, por consequência, a diferenciação no mercado.

\section{GESTÃO DO CONHECIMENTO}

A criação de conhecimento organizacional é compreendida como a habilidade de uma organização em criar conhecimento novo, de expandi-lo e de agrupá-lo a produtos, a serviços e a sistemas na organização como um todo, proporcionando o seu aprimoramento contínuo (NONAKA; TAKEUCHI, 1997). Ele é resultado de uma ação em movimento contínuo que se inicia através da aquisição e interação das pessoas com a informação, as quais potencializam e geram conhecimentos através das interpretações e do desenvolvimento de habilidades e competências (BRAUN; MUELLER, 2014).

A GC tem como princípio fundamental a gestão do capital humano e intelectual. Busca compreender as características do ambiente competitivo e as necessidades coletivas e individuais, refletindo a coordenação sistemática de esforços nos níveis operacional e 
estratégico (TERRA, 2014; VALENTIM, 2003).

Tendo em vista que o conhecimento circulante nas organizações representa o principal ativo garantidor da sobrevivência dos negócios na esfera atual do mercado (BRAUN; MUELLER, 2014; MENEZES et al., 2017; SANTIAGO JR, 2014), objetiva a aplicação de estratégias com enfoque cognitivo (VALENTIM, 2003). O conhecimento dissipado cria um modelo mental para toda a organização, de forma que as ações e decisões são modeladas em comportamentos compartilhados por todos os indivíduos, com impacto na aprendizagem desses (REZENDE, 2003; TERRA, 2014).

A GC apresenta as seguintes vantagens: 1) produz resposta às demandas no mercado; 2) gera lucratividade; 3) fomenta produtividade; e 4) reduz custos do empreendimento. Dessa maneira, promove a transformação de informações internas fragmentadas em representações com significados e estruturas, acionando ações corretivas em casos de falhas e identificando oportunidades de mercado, sendo assim a base para a inteligência competitiva nas organizações (RIBEIRO et al., 2017; SANTIAGO JR., 2014; TERRA, 2014; VALENTIM, 2003).

Para realizar a GC, é necessário focar tanto na dimensão tácita (comportamental) como na dimensão explícita (estrutura e tecnologia) de modo a gerar efetividade organizacional (GARCIA; COLTRE, 2017). Ela representa uma abordagem deliberada e sistemática que utiliza o conhecimento organizacional vinculado às habilidades individuais, competências, pensamentos, inovações e ideias com o objetivo de criar uma empresa mais eficiente e efetiva (CENTENARO; BONEMBERGER; LAIMER, 2016).

Nesse sentido, no âmbito da GC, é necessário atentar para aspectos como: 1) enquanto os colaboradores focam no aprendizado, a alta administração na definição dos campos do conhecimento; 2) ela demanda novas práticas de organização do trabalho e novas estruturas organizacionais; 3) tem foco nos ativos intangíveis (homem); 4) busca a criação de ferramentas e a prática de incentivos para o compartilhamento de conhecimento; 5) envolve o emprego de tecnologias para comunicação interna entre colaboradores (intranet, groupware etc.); 6) demanda mapeamento do conhecimento (TERRA, 2014).

Para tanto, é preciso que exista a definição prévia da estratégia para o aproveitamento do "patrimônio intelectual" e o monitoramento e avaliação constantes de dados com vistas ao manuseio de informações e conhecimentos (VALENTIM, 2003). A GC, então, por não estar materializada na estrutura organizacional, possibilita a criação de vantagem competitiva e dificulta a imitação por empresas concorrentes (RIBEIRO et al., 2017; SILVA, 2004).

No âmbito da GC pode-se entender, então, que a cognição é a principal habilidade das organizações, contribui para a administração de recursos estratégicos, o desenvolvimento de competências essenciais e a criação de vantagem competitiva sustentável (NOBRE; TOBIAS; WALKER, 2011). 


\section{DISCUSSÃO}

A pesquisa empreendida apontou usos e aplicações da TI, SIG e GC enquanto ferramentas de gestão empresarial contemporâneas.

Pode-se perceber que a introdução de uma TI, um SIG ou de uma GC promove mudanças na forma de funcionamento da empresa (PRATES; OSPINA, 2004), afetando seus processos de trabalho. Em função disso, a utilização destas ferramentas pode sofrer, inicialmente, recusa ou oposição por parte dos colaboradores.

Percebeu-se, contudo, que com o passar dos tempos as ferramentas evoluíram e passaram a exercer funções mais estratégicas (SILVA, 2002). O que pode ser explicado pela difusão de modelos de gestão mais adequados aos cenários competitivos contemporâneos, como o New Industrial Organization, por exemplo (BRITO; BRITO, 2012).

Foi possível observar que os usos da TI são relacionados a transformação de dados em informações confiáveis e atualizadas com vistas à solução de problemas e à tomada de decisões (RODRIGUES; PINHEIRO, 2005). Tal fato sugere que a busca pela vantagem competitiva pode perpassar pela implementação de uma TI.

Outro fato interessante diz respeito as associações das ferramentas TI e SIG ao planejamento estratégico das empresas. A TI postula a necessidade do sinergismo e a coordenação ao longo do tempo (LAURINDO et al., 2001; LUZ et al., 2016). Os SIG possibilitam aos seus usuários atividades de planejamento, desenvolvimento e proposição de soluções para problemas operacionais, gerenciais ou estratégicos (REZENDE; ABREU, 2006). A sinergia entre ambos é explicada pelo fato de que os SIG são resultados da implementação da TI (BALARINE, 2002). Assim, não há como dissociar os SIG da TI.

Percebeu-se que a GC se inicia através da aquisição e interação das pessoas com as informações (BRAUN; MUELLER, 2014) e que a mesma tem como princípio fundamental a gestão do capital humano e intelectual (TERRA, 2014; VALENTIM, 2003). Tal fato pode ser explicado pela grande relevância das pessoas enquanto recursos estratégicos na atual sociedade do conhecimento.

Observou-se a associação da GC enquanto recurso para a compreensão das características do ambiente e das necessidades coletivas e individuais (TERRA, 2014; VALENTIM, 2003), o que pode ser compreendido como fator essencial para o desenvolvimento sustentável das organizações (PORTER, 1989).

Além disso, a literatura têm associado as vantagens do uso da GC a produção de respostas às demandas no mercado, geração de lucro, fomento à produtividade e redução dos custos dos empreendimentos (RIBEIRO et al., 2017; SANTIAGO JR., 2014; TERRA, 2014; VALENTIM, 2003), aos ganhos relacionados a influência da GC no desempenho e na efetividade organizacional (CENTENARO; BONEMBERGER; LAIMER, 2016) e entre capital humano e capital 
estrutural na vantagem competitiva da empresa (OLIVEIRA FILHO; LOPES; OLIVEIRA, 2014). Fatores estes considerados garantidores da sobrevivência dos negócios no mercado atual (BRAUN; MUELLER, 2014; MENEZES et al., 2017; SANTIAGO JR, 2014).

Estudos recentes apontam resultados positivos entre a GC e a vantagem competitiva (CENTENARO; BONEMBERGER; LAIMER, 2016; OLIVEIRA FILHO; LOPES; OLIVEIRA, 2014). Entende-se que a GC pode influenciar o desempenho e a efetividade organizacional (CENTENARO; BONEMBERGER; LAIMER, 2016). As organizações que possuem ambiente favorável para a criação, uso e compartilhamento do conhecimento tácito e explícito têm mais possibilidades de sucesso na obtenção de vantagem competitiva frente aos concorrentes (CENTENARO; BONEMBERGER; LAIMER, 2016). Além disso, resultados recentes, revelam fortes influências positivas entre capital humano e capital estrutural na vantagem competitiva da empresa (OLIVEIRA FILHO; LOPES; OLIVEIRA, 2014).

Para a implementação destas ferramentas fica evidente a importância de se conhecer a empresa, através de sua estrutura, recursos e processos. A inserção da empresa no mercado, seus objetivos, metas e estratégias devem ser levantados para verificar em que medida elas buscam ou necessitam de vantagens competitivas. Vale lembrar, nesse sentido, que tanto os elementos materiais quanto os imateriais (simbólicos), precisam ser apreendidos, de modo a favorecer potencialidades e minimizar as dificuldades da TI, SIG e GC em cada organização.

Tais constatações parecem estar associadas aos ideais da vantagem competitiva, uma vez que os mesmos têm por objetivo a promoção de respostas adaptativas das organizações ao ambiente em que elas estão inseridas, através de competências e recursos valiosos, raros, inegociáveis e insubstituíveis (ALVARENGA et al., 2015; BARNEY, 1991; NOBRE; TOBIAS; WALKER, 2011).

Assim, defende-se que empresas façam o uso adequado e inteligente dos recursos disponíveis com vistas à criação de estratégias que levem ao aproveitamento de oportunidades. A criação de vantagens competitivas, por meio de ferramentas como TI, SIG e GC figura, então, como caminho para o posicionamento vantajoso em mercados de elevada concorrência.

\section{CONSIDERAÇÕES FINAIS}

O fluxo de informações funciona como o elemento unificador entre o uso de computadores, tecnologias, hardwares e serviços (TI) e sistemas de informações, os quais facilitam tomadas de decisão (SIG) e formas de manuseio da tecnologia e das pessoas em nível operacional e estratégico (GC).

Entende-se que a TI, os SIG e a GC convergem atuando como ferramentas de gestão, atuando para o alcance dos objetivos organizacionais. Muito embora essas três ferramentas sejam de aplicação individual, com usos, vantagens e desvantagens específicos, é notável que 
a integração das mesmas possibilita melhores resultados, com repercussões positivas para a criação de vantagens competitivas e para o sucesso da empresa.

Esta pesquisa contribui para o desenvolvimento organizacional uma vez que apresenta a compilação de temas relevantes ligados aos estudos da gestão e negócios, os quais ainda precisam ser mais explorados, tanto no ambiente acadêmico, quanto nas práticas empresariais. Portanto, sugere-se a realização de pesquisas empíricas sobre a relação destes conceitos.

Em se tratando de uma pesquisa do tipo revisão narrativa de literatura destaca-se como limitação o fato de que as escolhas dos estudos (livros e artigos) e a interpretação das informações podem estar sujeitas à subjetividade dos autores. Assim, de modo a ampliar esta perspectiva, estimula-se o desenvolvimento de pesquisas de cunho bibliométrico, em base de dados internacionais, que possam oferecer um panorama sobre a evolução dos temas, as principais linhas de estudo, formatos de pesquisas, dentre outras informações, que possam ser úteis para propor uma agenda de pesquisa sobre estes temas.

\section{REFERÊNCIAS}

ALBERTIN, A. L. Aumentando as chances de sucesso no desenvolvimento e implementação de sistemas de informações. Revista de Administração de Empresas, São Paulo, v. 36, n. 3, p. 6169, 1996.

ALMEIDA, M. V.; MARCONDES, R. C. A distribuição física como recurso estratégico de fabricantes de bens de consumo para a obtenção da vantagem competitiva. Revista de Administração, São Paulo, v. 49, n. 4, p. 656-670, 2014.

ALVARENGA, M. A. et al. Capacidades dinâmicas e vantagem competitiva em ambientes de mudanças constantes, à luz da análise do filme 'Recém-chegada'. Revista de Gestão, São Paulo, v. 24, n. 1, p. 35-44, 2017.

AUDY, J. L. N.; BECKER, J. L.; FREITAS, H. Modelo de planejamento estratégico de sistemas de informações: a visão do processo decisório e o papel da aprendizagem organizacional. In:

Encontro Nacional dos Programas de Pós-graduação em Administração, 23, p. 1-24, 1999.

BALARINE, O. F. O. Tecnologia da informação como vantagem competitiva. Revista de Administração de Empresas, São Paulo, v. 1, n. 1, p. 1-11, 2002.

BARNEY, J. B. Firms resources and sustained competitive advantage. Journal of Management, Thousand Oaks, v. 17, n. 1, p. 99-120, 1991.

BAZZOTTI, C.; GARCIA, E. A importância do sistema de informação gerencial para tomada de decisões. Ciências Sociais Aplicadas em Revista, Marechal Cândido Rondon, v. 6, n. 11, p. 1-18, 2006.

BRAUN, C. A.; MUELLER, R. R. A gestão do conhecimento na administração pública municipal 
em Curitiba com a aplicação do método OKA — Organizational Knowledge Assessment. Revista de Administração Pública, Rio de Janeiro, v. 48, n. 4, p. 983-1006, 2014.

BRITO, R. P.; BRITO, L. A. L. Vantagem Competitiva, Criação de valor e seus efeitos sobre o desempenho. Revista de Administração de Empresas, São Paulo, v. 52, n. 1, p. 70-84, 2012.

BRITO, R. P.; BRITO, L. A. L. Vantagem competitiva e sua relação com o desempenho: uma abordagem baseada em valor. Revista de Administração Contemporânea, Rio de Janeiro, v. 16, n. 3, p. 360-380, 2012.

CARMO, V. B.; PONTES, C. C. C. Sistemas de informação gerenciais para programa de qualidade total em pequenas empresas da região de Campinas. Ciência da Informação, Brasília, v. 28, n. 1, p. 49-58,1999.

CENTENARO, A.; BONEMBERGER, A. M. O.; LAIMER, C. G. Gestão do conhecimento e vantagem competitiva: estudo no setor metalmecânico. Revista de Ciências da Administração, Florianópolis, v. 18, n. 44, p. 38-51, 2016.

CONTO, S. M.; ANTUNES JÚNIOR, J. A. V.; VACCARO, G. L. R. A inovação como fator de vantagem competitiva: estudo de uma cooperativa produtora de suco e vinho orgânicos. Gestão \& Produção, São Carlos, v. 23, n. 2, p. 397-407, 2016.

CINTRA, P. F. et al. Impacto da implantação de um sistema de informação gerencial na gestão de contratos públicos: o caso do Hospital Universitário de Dourados/MS. Revista de Administração da UNIMEP, Piracicaba, v. 10, n. 2, p. 28-52, 2012.

DE HAES, S.; VAN GREMBERGEN, W.; DEBRECENY, R. S. COBIT 5 and enterprise governance of information technology: building blocks and research opportunities. Journal of Information Systems, Lakewood Ranch, v. 27, n.1, p. 307-324, 2013.

DEBRECENY, R. S.; GRAY, G. L. IT Governance and process maturity: a multinational field study. Journal of Information Systems, Lakewood Ranch,v. 27, n. 1, p. 157-188, 2013.

GARCIA; O. P. G.; COLTRE, S. M. A gestão do conhecimento como fator determinante na retenção dos colaboradores na empresa: um estudo de caso em uma organização do ramo moveleiro. Brazilian Business Review, Vitória, v. 14, n. 2, p. 182-203, 2017.

GHEMAWAT, P. A estratégia e o cenário dos negócios. Porto Alegre: Bookman, 2007.

GIL, A. C. Como elaborar projetos de pesquisa. 4ạ ed. São Paulo: Atlas, 2002.

ITO, N. C., HAYASHI, P., GIMENEZ, F. A. O. FENSTERSEIFER, J. E. Valor e vantagem competitiva: buscando definições, relações e repercussões. Revista de Administração Contemporânea, Rio de Janeiro, v. 16, n. 2, p. 290-307, 2012.

JUNIOR, S. S.; FREITAS, H. M. R.; LUCIANO, E. M. Dificuldades para o uso da tecnologia da informação. Revista de Administração de Empresas, São Paulo, v. 4, n. 2, p. 1-25, 2005. 
LAURINDO, F. J.; SHIMIZU, T.; CARVALHO, M. M.; JR, R. R. O papel da tecnologia da informação (TI) na estratégia das organizações. Gestão \& Produção, São Carlos, v. 8, n. 2, p.160-179, 2001.

LUZ, T. A. et al. Avaliação de desempenho de serviços de tecnologia da informação: identificação do estado da arte por meio de um processo de pesquisa construtivista e análise bibliométrica. Perspectivas em Ciência da Informação, Belo Horizonte, v. 21, n. 2, p.120-140, 2016.

MINAYO, M. C. S. Pesquisa social: teoria, método e criatividade. Petrópolis: editora vozes, 2008.

MENEZES, K. C. et al. Gestão do conhecimento nas organizações: uma aprendizagem em rede colaborativa. Perspectivas em Gestão \& Conhecimento, João Pessoa, v. 7, n. 1, p. 145-159, 2017.

MORAES, G. D. A.; TERENSE, A. C. F.; FILHO, E. E. A tecnologia da informação como suporte à gestão estratégica da informação na pequena empresa. Revista de Gestão da Tecnologia e Sistemas de Informação, São Paulo, v. 1, n. 1, p. 27-43, 2004.

NOBRE, F. S.; TOBIAS, A. M.; WALKER, D. S. Uma visão da empresa baseada em habilidades: contextos estratégicos e contingenciais. Revista de Administração Contemporânea, Curitiba, v. 15, n. 3, p. 413-432, 2011.

NONAKA, I; TAKEUCHI, H. Criação do conhecimento na empresa. Rio de Janeiro: Campus, 1997.

PORTER, M. E. Competitive advantage: creating and sustaining superior performance. New York: Free Press, 1985

PORTER, M. E. Vantagem competitiva: criando e sustentando um desempenho superior. Rio de Janeiro: Elsevier, 1989.

OLIVEIRA, D. P. R. Sistemas de informação gerenciais: estratégias, táticas, operacionais. 8. ed., São Paulo: Atlas, 1992.

OLIVEIRA FILHO, J. B.; LOPES, J. E. F.; OLIVEIRA, M. F. O efeito da gestão do capital intelectual na vantagem competitiva: o caso de um grupo empresarial com atuação predominante em tecnologia. Revista Gestão Organizacional, Chapecó, v. 7, n. 2, p. 89-101, 2014.

PEREZ, G. Adoção de inovações tecnológicas: Um estudo sobre o uso de sistemas de informação na área de saúde. Tese (Doutorado em Administração) - Universidade de São Paulo, São Paulo, 2006.

PRATES, G. A.; OSPINA, M. T. Tecnologia da informação em pequenas empresas: fatores de êxito, restrições e benefícios. Revista de Administração Contemporânea, v. 8, n. 2, p. 9-26, 2004. 
RIBEIRO, J. S. A. N. et al. Gestão do conhecimento e desempenho organizacional: integração dinâmica entre competências e recursos. Perspectivas em Gestão \& Conhecimento, João Pessoa, v. 7, n. 1, p. 4-17, 2017.

REZENDE, D. A.; ABREU, A. F. Tecnologia da informação aplicada a sistemas de informação empresariais. São Paulo: Atlas, v. 3, 2000.

REZENDE D. A.; ABREU, A. F. Tecnologia da Informação: Aplicada a Sistemas de Informação Gerenciais. Atlas, 2006.

REZENDE, S. O. Sistemas inteligentes: fundamentos e aplicações. São Paulo. Manole, 2003.

RODRIGUES, E.; PINHEIRO, A. S. Tecnologia da informação e mudanças organizacionais. Universidade IMES. São Caetano do Sul (SP). 2005.

RODRIGUES, E.; PINHEIRO, M. A. S. Tecnologia da informação e mudanças organizacionais. Revista de Informática Aplicada, São Caetano do Sul, v. 1, n. 2, p. 101-112, 2010.

ROSSETTI, A. G.; MORALES, A. B. T. O papel da tecnologia da informação na gestão do conhecimento. Ciência da Informação, Brasília, v. 36, n. 1, p. 124-135, 2007.

ROTHER, E. T. Revisão sistemática x revisão narrativa. Acta Paulista de Enfermagem, São Paulo, v. 20, n. 2, p. 5-6, 2007.

SANTIAGO JR, J. R. S. Gestão do conhecimento. São Paulo: Novatec Editora, 2014.

SILVA, S. L. da. Informação e competitividade: a contextualização da gestão do conhecimento nos processos organizacionais. Ciência da Informação, Brasília, v. 31, n. 2, p. 142-151, 2002.

SILVA, S. L. Gestão do conhecimento: uma revisão crítica orientada pela abordagem da criação do conhecimento. Ciência da Informação, Brasília, v. 33, n. 2, p. 143-151, 2004.

STAIR, R. M. Princípios de sistemas de informação: uma abordagem gerencial. Rio de Janeiro: LTC, 1998.

TERRA, J. C. C. (s/f). Gestão do Conhecimento: O grande desafio empresarial! Biblioteca Terra. Fórum Consultores, 2014.

VASCONCELOS, F. C.; CYRINO, A. B. Vantagem competitiva: os modelos teóricos atuais e a convergência entre estratégia e teoria organizacional. Revista de Administração de Empresas, São Paulo, v. 40, n. 4, p. 20-37, 2000.

VALENTIM, M. L. P. et al. O processo de inteligência competitiva em organizações. Data Grama Zero, Rio de Janeiro, v. 4, n. 3, p. 1-23, 2003.

VIANA, F. L. E.; NETO, J. D. P. B.; AÑEZ, M. E. M.; FERNANDES, J. A. L. Fontes de obtenção de vantagem competitiva em empresas industriais: uma análise nas indústrias têxtil e de calçados do Ceará. Revista Econômica do Nordeste, Fortaleza, v. 43, n. 3, p. 533-560, 2016. 